\title{
Evaluación de la calidad total en servicios municipales deportivos orientados a la población infantil: Aportaciones desde el análisis cualitativo con ATLAS.ti
}

\author{
Evaluation of total quality in sports municipal services geared to \\ children: Contributions from the qualitative analysis ATLAS.ti
}

\section{Avaliação da qualidade total em serviços municipais de desporto dirigidos á população infantil: Contribuiçóes da análise qualitativa com ATLAS.ti}

\author{
Rocío Pérez-López ${ }^{1}$, Verónica Morales-Sánchez ${ }^{1 *}$, M. Teresa Anguera² y Antonio Hernández-Mendo ${ }^{1}$ \\ ${ }^{1}$ Universidad de Málaga, ${ }^{2}$ Universidad de Barcelona, España
}

Resumen: El presente trabajo tiene como objetivo evaluar la calidad total de los servicios deportivos municipales de Alcalá la Real (Jaén), analizando tres dimensiones: funcional, emocional y aspectos tangibles. La muestra estuvo formada por usuarios/as infantiles junto con los gerentes, el personal de contacto (atención al usuario/a y monitores/as) de las instalaciones municipales deportivas, el coordinador del Área de Deportes del Ayuntamiento y padres/madres de los/las nińos/as que participan en las diferentes actividades deportivas. La metodología empleada es cualitativa a través del análisis de contenido que se realizó con el programa Atlas.ti 7.1.3. Los instrumentos de recogida de información fueron entrevistas individuales y grupos focales grabadas con cámaras digitales. Se concluye que los/las usuarios/as infantiles al evaluar la calidad total de los servicios deportivos dan una relevancia importante a los aspectos tangibles, y destacan los aspectos emocionales y de relación afectiva sobre los funcionales de la calidad del servicio.

Palabras Clave: Gestión deportiva, calidad total, calidad funcional, calidad emocional, aspectos tangibles.

Abstract: The aim of the current study is to assess the total quality of the municipal sports services of Alcalá la Real, analyzing the three dimensions of quality service: functional, emotional and tangibles. The sample is composed of child users, contact personnel (attention personnel of the users and coaches) of the municipal sports facilities, sport coordinator of the town council and the parents of the children the different sports. The qualitative methodology has used for the content analysis. The instrument used for data collections are individual interviews and focus groups filmed with a video camera. For content analysis we used the Atlas.ti 7.1.3 software. According to results tangibles were very important for child users when they evaluat the total quality of the sports services and the emotional aspect were also found to me more important than the functional quality of service. Key words: Sports management, total quality, functional quality, emotional quality, tangible aspects.

Resumo: Este estudo tem como objetivo avaliar a qualidade geral dos serviços desportivos municipais de Alcalá la Real (Jaén), analisando três dimensōes: funcional, emocional e aspectos tangíveis. A amostra foi constituída por utilizadores(as) crianças, juntamente com os gestores, o pessoal de contacto (atendimento ao cliente e monitores ( as), o coordenador da área de Desporto do Município e pais/mães de crianças que participam nas diferentes actividades desportivas. A metodologia utilizada é qualitativa recorrendo-se à análise de conteúdo, que foi realizada com o programa Atlas.ti 7.1.3. Os instrumentos de recolha de informação utilizados foram entrevistas individuais e a grupos focais gravadas com câmeras digitais. Conclui-se que os utilizadores/crianças ao avaliarem a qualidade total dos serviços desportivos dão uma relevância importante aos aspectos tangíveis, e enfatizam os aspectos emocionais e afetivos relativamente aos aspectos da qualidade funcional do serviço.

Palavras Chave: Gestão desportiva, qualidade total, qualidad funcional, qualidade emocional, aspectos tangíveis.

\section{Introducción}

En las organizaciones deportivas la calidad total del servicio es una estrategia que les permite diferenciarse, y por la que adquieren un alto compromiso con sus usuarios/as ya que su finalidad es satisfacer las necesidades, preocupaciones, aportaciones, y requerimientos de la ciudadanía, como es en el caso de los servicios municipales.

La calidad total es una filosofía empresarial coherente

Dirección para correspondencia [Correspondence address]: Verónica Morales-Sánchez. Depto. Psicología Social, Antropología Social, Trabajo Social y Servicios Sociales. Facultad de Psicología. Campus de Teatinos, s/n. Universidad de Málaga. 29071 Málaga (Espańa).E-mail: vomorales@ uma.es orientada a satisfacer de manera permanente las necesidades y expectativas de los/las usuarios/as internos y externos. Por ello, la organización debe estar mejorando continuamente, con una participación activa y con el compromiso de todos/ as los/las integrantes de la organización. De este modo se logrará alcanzar un aumento del nivel de calidad, que persigue la excelencia, y que va a contribuir en la mejora de vida de los/ las ciudadanos/as.

Entre la población, se presta atención principalmente a la población infantil, debido a la importancia y los beneficios de la actividad física y el deporte en estas edades. En las primeras etapas del desarrollo, la actividad física juega un papel 
importante en el desarrollo físico (Corbin y Pangrazi, 2003; Malina y Bouchard, 1991), social (Committe on Sports Medicine and Fitness Comittee on School Health, 2001) y cognitivo de los jóvenes (Weinberg y Gould, 1996; Ntoumanis, 2001).

Para la evaluación de la calidad del servicio, es importante estimar las dimensiones a las cuales los/las usuarios/as dan mayor relevancia. En este estudio se parte de las dimensiones de la calidad del servicio del Modelo de Deficiencias, utilizando como base las cinco dimensiones que componen la herramienta SERVQUAL (Zeithaml, Parasuraman, y Berry, 1993): (a) elementos tangibles, definida como la apariencia de las instalaciones físicas, equipos, personal y materiales de comunicación; (b) fiabilidad, habilidad para realizar el servicio prometido de forma fiable y cuidadosa; (c) capacidad de respuesta, disposición y voluntad para ayudar a los/las usuarios/ as y proporcionar un servicio rápido; (d) seguridad, conocimientos y atención mostrados por los/las empleados/as y sus habilidades para inspirar credibilidad y confianza y (e) empatía, atención individualizada que ofrecen las empresas a sus consumidores.

Cuatro de estas cinco dimensiones se referían a la interacción entre empleado/a de contacto y usuario/a (fiabilidad, capacidad de respuesta, seguridad y empatía), y una referente a aspectos tangibles del servicio.

El modelo SERVQUAL fue empleado por Parasuraman, Zeithaml y Berry (1988) en diferentes tipos de servicios (servicios de mantenimiento, banca, uso de tarjetas de crédito, servicios telefónicos y negociación de valores). Dado que en estos servicios estudiados, el/la usuario/a tiene una visión muy superficial de lo tangible, es lógico que la dimensión de aspectos tangibles, tuviese una escasa valoración por los/las usuarios/as, considerándolo un aspecto secundario. De hecho, Parasuraman, Ziethaml y Berry (1985) observaron que esta dimensión era la menos importante para los/las usuarios/as y asumieron que la interacción entre profesional y usuario/a era predominante. No obstante, estudios posteriores, donde el/la usuario/a tiene un conocimiento profundo de las instalaciones (servicios deportivos, hospitales, etc.), se incrementa la importancia de los aspectos tangibles y físicos (Mañas, Jiménez, Mayor, Martínez-Tur y Moliner, 2008; Martínez-Tur, Peiró y Ramos, 2005; Richard y Sundaramen, 1994)

Debido a que este modelo se concentra especialmente en los aspectos funcionales de la interacción del usuario/a con las personas de contacto y con la organización, tras un estudio piloto realizado y al tratarse de población infantil, se estimó la necesidad de ampliar las categorías para poder reflejar los aspectos más emocionales o relacionales.

Para delimitar estos aspectos más relacionales o emocionales del encuentro de servicio, Price, Arnould y Tierney (1995) se refirieron a los conceptos de comprensión auténtica y extras o iniciativa. Se entiende la comprensión auténtica como la capacidad de los trabajadores/profesionales de contacto para "sintonizar" con las vivencias y sentimientos de los/las usuarios/as y ser genuinos en la atención, conectar con la vida de los/las usuarios/as. Los extras o iniciativa, son los esfuerzos que realizan dichos trabajadores y profesionales para dar un servicio excelente. Generan sorpresa porque superan lo estándar, lo habitual, o lo que es formal en un contrato.

Estudios recientes, recogen conceptos como aspectos tangibles, calidad del servicio funcional, relacional o emocional (Sánchez-Hernández, Martínez-Tur, Peiró y Ramos, 2009). La calidad de servicio funcional (eficiencia en atender a los usuarios en los aspectos centrales del servicio) incluía ítems tradicionalmente considerados en la escala SERVQUAL con respecto a las dimensiones de interacción del usuario con la organización y sus trabajadores. Esta dimensión general se articulaba a partir de las cuatro dimensiones de la escala SERVQUAL (fiabilidad, seguridad, capacidad de respuesta y empatía), sustituyendo la etiqueta de "empatía" por la de "atención individualizada".

En la escala SERVQUAL se define la empatía desde una perspectiva muy funcional que hace referencia a la atención individualizada al/a usuario/a. En cambio, el concepto de empatía es más complejo (Baron-Cohen y Wheelwright, 2004), y contiene un componente emocional más allá de un servicio individualizado. Sánchez-Hernández, Martínez-Tur, Peiró y Ramos (2009), definen la empatía, en los encuentros de servicios, como el grado en que los trabajadores y profesionales son capaces de ponerse en el lugar de los/las usuarios/as, experimentando en cierta medida las emociones de dichos/as usuarios/as como si fueran las suyas propias y ofreciendo un beneficio que no puede abordarse desde la óptica tradicional de la calidad de servicio funcional (p.e. la empatía del trabajador ante una queja del usuario). Por lo tanto se puede apreciar una evolución del concepto de empatía hacia una perspectiva emocional/relacional en la calidad del servicio.

De esta manera, para entender la importancia que se otorga al estudio de las emociones de los participantes en la comprensión de la satisfacción de los/las usuarios/as se ha considerado imprescindible recoger las tres dimensiones descritas, partiendo de distintos modelos conceptuales hasta centrase en uno de los objetivos específicos "la calidad emocional/relacional".

La calidad del servicio relacional o emocional se materializa en aquellos beneficios relacionales y emocionales que el/ la usuario/a recibe más allá del servicio nuclear propiamente dicho (Gwinner, Gremler y Bitner, 1998); Peiró, MartínezTur y Ramos, 2005). Incorpora ítems relativos a dimensiones más específicas de calidad que tiene que ver con los beneficios emocionales: comprensión auténtica, extras y empatía. Los aspectos tangibles (instalaciones y equipamiento) formaban la tercera dimensión general de la calidad del servicio.

El principal objetivo de estudio es evaluar la calidad total de 
los servicios deportivos orientados a la población infantil y contribuir a conocer sus necesidades, expectativas y percepciones sobre los servicios deportivos recibidos, que es tan importante para la mejora de la satisfacción de los/las usuarios/as. De este modo, se pretende mejorar la calidad de los servicios deportivos y transmitir la importancia de la adherencia y consolidación de hábitos de práctica deportiva desde las primeras edades de los/las niños/as, repercutiendo en estilos de vida saludable.

\section{Método}

\section{Participantes}

Se obtuvo una muestra de $74(\mathrm{n}=74)$ participantes a través de un muestreo intencional, de los cuales 47 participaron en las entrevistas individuales y 27 en los grupos focales.

De los 47 participantes de las entrevistas en profundidad, 29 usuarios/as tienen un rango de edad de 5 a 12 ańos, y además hay 7 padres/madres, 6 monitores, 2 personas de atención al usuario, 2 gerentes y 1 coordinador del Área de Deportes del Excmo. Ayuntamiento.

Los integrantes de los grupos focales se distribuyeron en tres sesiones, la primera de ellas formada por 9 participantes con edades comprendidas entre los 5 y 8 años. Una segunda sesión con 8 usuarios/as con un rango de edad entre 9 y 12 años. Y la tercera sesión estuvo constituida por representantes de todos los ámbitos a evaluar; 2 gerentes, 1 coordinador, 1 persona de atención al usuario, 2 monitores, 2 madres y 2 nińos.

Previo a la grabación de las sesiones a los/las usuarios/as infantiles se obtuvo el correspondiente consentimiento informado debidamente firmado por los padres de acuerdo con la declaración de Helsinki (Rothman, Michels y Baum, 2000).

\section{Instrumentos}

A partir de los indicadores de la escala de SERVQUAL se elaboraron el guión de dos entrevistas en profundidad e individuales para los 47 participantes y estructuradas para los grupos focales. Ambos tipos de entrevistas se estructuraron con preguntas referidas a las actividades deportivas, el/la monitor/a, el material, la instalación deportiva y la organización.

En la recogida y grabación de la información de las entrevistas individuales y grupos focales se utilizó una cámara digital Sony HDR-CX505VE. Para el análisis cualitativo se empleó el programa ATLAS.ti versión 7.1.3.

\section{Procedimiento}

En la elaboración de las preguntas de las entrevistas individuales y de los grupos focales se partió, en primer lugar, de los cinco criterios del modelo SERVQUAL (elementos tangibles, fiabilidad, capacidad de respuesta, seguridad y empatía). En- tre las numerosas herramientas de evaluación en la literatura científica, se tomó como punto de partida las investigaciones de Morales-Sánchez (2003) y Morales-Sánchez, HernándezMendo y Blanco $(2005,2009)$, donde evalúan la calidad de los servicios municipales deportivos a través de la realización de una adaptación del modelo SERVQUAL para las organizaciones de servicios deportivos (Parasuraman, Zeithaml y Berry, 1988, 1993).

Posteriormente, como ya se ha comentado, se tuvo especial interés en la evaluación de elementos emocionales, por ello fue necesario ajustar estas dimensiones al modelo tridimensional para poder evaluar los aspectos más emocionales o relacionales (Price, Arnould y Tierney, 1995; Sánchez-Hernández, Martínez-Tur, Peiró y Ramos, 2009).

De esta manera, para delimitar los aspectos emocionales, se recogieron los conceptos de "comprensión auténtica” y "extras" (Price, Arnould y Tierney, 1995) y finalmente se recogieron las dimensiones de la calidad, a las que se refirieron, Sánchez-Hernández, Martínez-Tur y Ramos (2009): funcional, emocional/relacional y aspectos tangibles.

Finalmente, se establecieron las dimensiones del presente estudio del siguiente modo; en la calidad funcional se analizó con aspectos como la fiabilidad, seguridad, capacidad de respuesta, en la calidad emocional o relacional se tuvo en cuenta la empatía, comprensión auténtica y extra o iniciativa; en los aspectos tangibles se reflejaron la calidad de las instalaciones y equipamientos, y la apariencia adecuada del personal de contacto.

Las entrevistas individuales estaban estructuradas de forma que en una primera parte se incluían datos sociodemográficos, y en una segunda parte, preguntas referidas a la actividad deportiva, al monitor, el material, las instalaciones, espacios deportivos y la organización.

En cuanto a los grupos focales, la primera parte incluye una introducción, donde en las sesiones enfocadas a la población infantil, se realizó una dinámica de juego para la presentación de los participantes, posteriormente se da a conocer el propósito de la sesión y las reglas utilizadas para llevar a cabo la misma. Seguidamente, se pasa al desarrollo del tema, aquí los objetivos de la investigación se convierten en preguntas.

Para el análisis de contenido, se utilizó el programa ATLAS.ti. v. 7.1.3, un programa informático de gran alcance para el análisis cualitativo de grandes cuerpos de datos textuales, gráficos, audio y vídeo (Morales-Sánchez, Pérez-López y Anguera, 2014).

Para analizar el contenido cualitativo de las entrevistas y los grupos focales, las grabaciones en vídeo de las mismas (documentos primarios) se han incorporado para el análisis en formato multimedia, habiéndose organizado y almacenado en un único archivo (Unidad Hermenéutica, Figura 1). En este archivo se encuentra toda la información producida en el transcurso del análisis, además de los documentos primarios, contiene los 
códigos, las anotaciones o memos, las familias de documentos primarios, las familias de códigos y las familias de memos.

Figura 1. Descriptivos del análisis del contenido

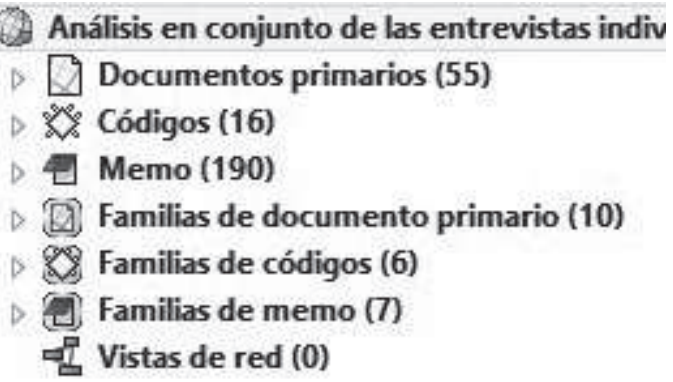

Los documentos primarios corresponden a cada una de las entrevistas y sesiones de grupos focales en grabaciones de vídeo y documentos de texto complementarios al estudio. Las citas son las segmentaciones o fragmentos de los vídeos que son más significativos de los documentos primarios y que pueden ser asociados a un código. Los códigos son los elementos principales del análisis y se pueden considerar como un sistema de categorías. Las anotaciones o memos, son todos aquellos comentarios, anotaciones que se han considerado importante recoger o aclarar.

La segmentación de la grabación de vídeo y su codificación es una forma de reducción de datos, puesto que, partiendo del gran volumen de información, se segmentan algunas partes de los documentos (citas), que a su vez pueden asociarse a los códigos. Además estos códigos se organizan en familias.

En el caso de los documentos primarios, en la Figura 1, se muestran agrupados en 10 familias. En la Figura 2, se puede ver una familia de códigos agrupados en 6 bloques, que corresponden a las tres dimensiones, correspondientes a la distinción entre los aspectos de la calidad del servicio positivos u óptimos frente a los de calidad negativa o no óptima.

Figura 2. Familias de códigos.

\begin{tabular}{|c|c|}
\hline 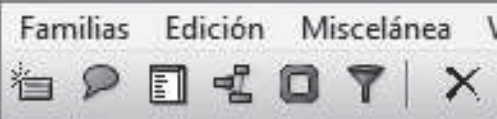 & $\begin{array}{l}\text { sualizar } \\
\text { 国 }\end{array}$ \\
\hline Nombre & Tamaño \\
\hline Q8spectos tangibles no óptimos & 2 \\
\hline Q. Aspectos tangibles óptimos & 2 \\
\hline (2) Calidad funcional no óptima & 3 \\
\hline 28 Calidad funcional óptima & 3 \\
\hline 28 Calidad relacional no óptima & 3 \\
\hline$\$$ Calidad relacional óptima & 3 \\
\hline
\end{tabular}

Los códigos están agrupados en seis familias que corresponden a las tres dimensiones de la calidad del servicio: Aspectos tangibles no óptimos, aspectos tangibles óptimos, calidad funcional no óptima, calidad funcional óptima, calidad relacional no óptima y calidad relacional óptima. (Figura 2).

\section{Resultados}

En el análisis de contenido de los documentos primarios (entrevistas individuales y grupos focales), la información se ha reducido tras la codificación, en citas que se asociaron a cada uno de los códigos. En la Figura 3 se aprecia como el código "Calidad adecuada, óptimas de las instalaciones y equipamientos" se ha codificado 208 veces, "Calidad no adecuada, no óptima de las instalaciones y equipamientos" tiene una frecuencia de 116, y de manera decreciente hasta el código "Apariencia no adecuada del personal de contacto" sin ninguna codificación.

Figura 3. Fundamentación de códigos. Calidad mala de las instalaciones y equipamientos.

\begin{tabular}{|c|c|c|}
\hline Nombre & \multicolumn{2}{|c|}{ Fundamentado } \\
\hline 2: Aparicencia adecuada del personal de contacto & in & 46 \\
\hline 2. Apariencia no adecuada del personal de contacto & & 0 \\
\hline 绪 Calidad no optima de las instalaciones y equipamientos & Elas & 116 \\
\hline Z Calidad optima de las instalaciones y equipamientos & E & 208 \\
\hline Capacidad de respuesta & in & 96 \\
\hline 渵 Comprensión auténtica & Enes & 112 \\
\hline * Empatía & $=$ & 100 \\
\hline 䍐 Fiabilidad & $1=$ & 147 \\
\hline 䄑 Iniciativa & I & 8 \\
\hline 2. Inseguridad & I & 23 \\
\hline No capacidad de respuesta & i & 32 \\
\hline No comprensión auténtica & 1 & 8 \\
\hline No No empatía & ii & 27 \\
\hline *: No fiabilidad & i & 14 \\
\hline No iniciativa & i & 2 \\
\hline 消 Seguridad & E & 55 \\
\hline
\end{tabular}

El código "Calidad no óptima de las instalaciones y equipamientos" tiene una frecuencia (fundamentación) de 116. No se debe al mal estado de la instalación, los pavimentos, campos de césped, sino a la limpieza. Los/las usuarios/as, por ejemplo, indican literalmente "Después del día del campeonato que fue el sábado, cuando llegamos estaba alli todo llenos de huellas, sucio....algunas veces, pero pocas, hay papel higiénico,...no hay jabón para lavarse las manos,... no hay papel para secarse." De modo que a través de los memos (Figura 4) se realiza una aclaración, anotando cual es el motivo de ese déficit.

Figura 4. Memo; no limpieza.

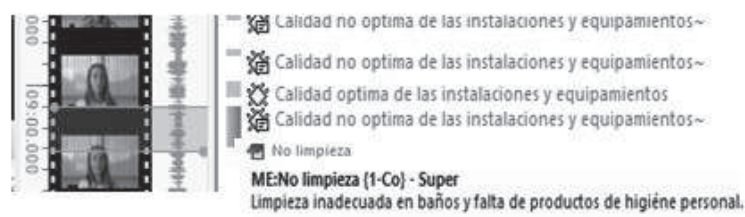


Los/las niños/as dan una elevada importancia al concepto de comprensión auténtica, como podemos ver en la Figura 3, en la que aparecen 112 citas asociadas al mismo. En la Figura 5, se presenta un ejemplo en que uno de los usuarios, nos trasmite: "Cuando voy a la piscina, me siento como si fuera mi casa porque los profesores son muy amables y como si fueran mis padres".

Figura 5. Comprensión auténtica.

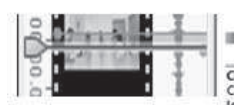

- Comprensión auténtica

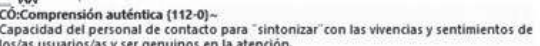

Otro de los usuarios comenta "Cita 1: Si alguna vez nos caemos y nos hacemos daño, él (monitor) nos cura" Cita 2: "Me siento muy tranquilo porque veo que me están vigilando y por si me pasa algo, que acuden a uno." (Figura 6).

Figura 6. Seguridad.

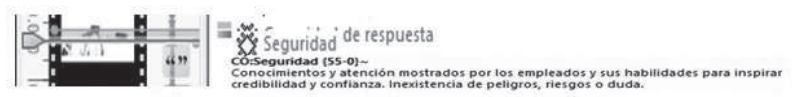

Los/las niños/as hablan de seguridad (conocimiento y atención individualizada mostrados por los empleados y sus ha- bilidades para inspirar credibilidad y confianza), con una concepción más allá del propio concepto, porque ellos/as hacen referencia a este término como una protección, la inexistencia de peligro, riesgo, cuando se les pregunta si se sienten seguros en el espacio deportivo en el que realizan su actividad deportiva.

Otro de los términos al que dan importancia tanto los adultos como los/las propios/as usuarios/as es fiabilidad, fundamentado con 147 citas. En la interacción del personal de contacto con los/las usuarios/as directos e indirectos, la fiabilidad de los servicios deportivos municipales es valorada positivamente.

Los aspectos de la calidad del servicio emocional, entre ellos comprensión auténtica, con 112 citas, es muy valorada por los/las usuarios/as. Los/las niños/as nos transmiten la importancia que para ellos/as tienen que su monitor/a principalmente, les traten bien, sean carińosos, simpáticos, etc. Para ellos un factor importante es el que se sientan bien y cómodos realizando su actividad deportiva. Además los/las padres/ madres realizan una valoración muy positiva del personal de las instalaciones deportivas, resaltando el buen trato de los/as monitores y monitoras hacia sus hijos/as y la profesionalidad de los mismos. Además algunos/as padres/madres destacan la cercanía del personal de contacto, expresando que hay una "relación familiar", dado el interés que muestran, que va más allá de lo normal en atender a los/las usuarios/as.

Figura 7. Tabla de co-ocurrencia de códigos.

\begin{tabular}{|c|c|c|c|c|c|c|c|}
\hline & Calidad mala & Capacidad de & Comprensión & Empatía & Fiabilidad & Iniciativa & Sequridad \\
\hline Aparicencia adecuada del & nas & $n / a$ & $n$ & $1-0,01$ & $n / 3$ & $n / a$ & $1-0,01$ \\
\hline Calidad buena de las insta & $10 \cdot 0,03$ & $1-0,00$ & $n / a$ & $1-0,00$ & $2-0,01$ & ñ/a & $3-0,01$ \\
\hline Capacidad de respuesta & na & & $3-0,01$ & $23-0,14$ & $6-0,03$ & $1-0,01 \quad 0$ & $6-0,04$ \\
\hline Comprensión auténtica & $n / a$ & $3-0,01$ & & $\bar{n} \bar{a}$ & $12-0,05$ & $n / a$ & $3-0,02$ \\
\hline Empatía & nas & $23-0,14$ & nua & & $8-0,03$ & $n / a$ & $1-0,01$ \\
\hline Fiabilidad & $n / a$ & $6-0,03$ & $12-0,05$ & $8-0,03$ & & 18 & $3-0,02$ \\
\hline Iniciativa & nia & $1-0,01$ & $n / a$ & ais & n/a & & rúa \\
\hline Seguridad & noa & $6-0,04$ & $3-0,02$ & $1-0,01$ & $3-0,02$ & $n / a$ & \\
\hline
\end{tabular}

Por último se realiza un análisis de co-ocurrencia (Figura 7), que permite visualizar en forma de "árbol o "tabla" la coocurrencia de códigos, es decir, aquellos códigos que están relacionados con "acotaciones similares". En la co-ocurrencia de los códigos; en fila observamos el código capacidad de respuesta y en columnas en código empatía. Su asociación estima datos positivos, con una frecuencia de 23 y un índice de 0,14 .

\section{Discusión}

Parasuraman (1999) en un estudio en el que participaron una empresa fabricante de ordenadores, una comercializadora, una aseguradora de autos y una aseguradora de vida, encon- 
tró que los clientes otorgan diferentes grados de importancia al momento de evaluar el servicio. El orden de influencia de estos atributos son: fiabilidad, capacidad de respuesta, seguridad, empatía y elementos tangibles. Los mismos resultados fueron encontrados en otros estudios acerca del SERVQUAL (Parasuraman, Zeithalm y Berry 1988, 1994) en los que la dimensión de aspectos tangibles tienen menos importancia para los/las usuarios/as de las organizaciones del servicio. Esto, es debido a que los/las usuarios/as prestan mayor importancia a otros aspectos como son la fiabilidad, capacidad de respuesta y no den mayor importancia a los aspectos tangibles.

En cambio, en estudios de evaluación de la calidad en organizaciones deportivas, entre ellos, el estudio realizado por Morales-Sánchez (2003) en la evaluación de las expectativas de los/las usuarios/as, se destacan por orden decreciente, como quedarían ordenados los factores de la calidad, medidos a través de la escala SERVQUAL, obteniendo como mejores resultados en los factores seguridad, capacidad de respuesta, empatía, elementos tangibles y fiabilidad.

Es importante destacar, que gran parte de los estudios están orientados a la evaluación sobre la calidad de los productos tangibles, y en los que se consideran algunos aspectos relevantes centradas en la calidad de los servicios (Grönroos, 1982; Berry, Shostack y Upah; 1983; Sasser, Olsen y Wychoff, 1978), como por ejemplo que para el usuario, evaluar la calidad de los servicios, es más difícil que evaluar la calidad de los productos tangibles. Es conveniente resaltar que los/las usuarios/as no sólo evalúan la calidad del servicio valorando el resultado final que reciben, sino que también toman en consideración el proceso de recepción del servicio y que en la evaluación de la calidad de un servicio, los únicos criterios que realmente son relevantes, son los que establecen los/las usuarios/as (Morales-Sánchez y Hernández-Mendo, 2004).

Los resultados estimados indican, que los/las usuarios/as dan una relevancia importante a la dimensión de aspectos tangibles, principalmente al atributo referido a la apariencia de las instalaciones físicas, material y limpieza.

Tal y como se ha señalado en el texto, es conveniente resaltar de estos resultados, que los/las usuarios/as infantiles en interacción con el personal de atención al usuario/a y monitores/as dan una mayor importancia a los atributos relacionados con los aspectos emocionales. Quedando evidenciado con la calidad óptima emocional presente, los/las usuarios/as nos transmiten directamente lo bien que se sientes "Tengo buen feeling con mi monitorla", "en la piscina me siento como si estuviera en mi casa", "mi monitorla me cura si me caigo y me hago una pupa", "en el polideportivo me siento seguro" (seguridad desde el punto de vista de protección).

De acuerdo con Gwinner, Gremler y Bitner (1998), los beneficios emocionales son especialmente evidentes cuando se alargan en el tiempo la relación entre empleado y cliente. Aunque también puede aparecer en interacciones de corta duración (Dubé y Menon, 1998).

En cualquier interacción de servicio, se exige al trabajador de contacto que sea capaz de llevar a cabo un adecuado trabajo emocional, mostrando aquellas emociones que se ajustan a las expectativas de los/las usuarios/as (Hochschild, 1983; Morris y Feldman, 1997; Zapf, Vogt, Seifert, Mertini y Isic, 1999).

\section{Aplicaciones Prácticas}

El presente estudio se centra en introducir mejoras conceptuales, metodológicas y tecnológicas en el proceso de evaluación de la calidad en organizaciones de servicios deportivos, concretamente los orientados a la población infantil y con ello, tratar de proponer un programa de intervención para la Mejora del Servicio orientado a la población Infantil.

Este trabajo forma parte de la investigación Observación de la interacción en deporte y actividad física: Avances técnicos y metodológicos en registros automatizados cualitativos-cuantitativos, que ha sido subvencionado por la Secretaría de Estado de Investigación, Desarrollo e Innovación del Ministerio de Economía y Competitividad [DEP2012-32124], durante el trienio 2012-2015

Convenio de colaboración entre la Universidad de Málaga (UMA) y el Excmo. Ayuntamiento de Alcalá la Real (Jaén), código de identificación no: 8.06/5.38.3668. Evaluación Psicosocial en Contextos Naturales: Deporte y Consumo (SEJ 444), financiado por la Junta de Andalucía (Consejería de Innovación, Ciencia y Empresa).

\section{Referencias}

1. Baron-Cohen, S. y Weelwright, S. (2004). The Empathy Quotient: An Investigation of Adults with Asperger Syndrome or High Functioning Autism, and Normal Sex Differences. Journal of Autism and Developmental Disorders, 34(2), 163-175.

2. Berry, Shostack y Upah, (1983). Relationship Marketing. In American Marketing Association, Emerging Perspectives on Service Marketing. Chicago: American Marketing Association.

3. Committee on Sports Medicine and Fitness and School Health. (2001). Organized sports for children and preadolescents. Pediatrics, 107(6), 1459-1462.
4. Corbin, C. B. y Pangrazi, R. P. (2003). Guidelines for appropriate physical activity for elementary school children: 2003 update. Reston, VA: NASPE Publications. Council on Physical Education for Children (COPEC). (1998). Physical Activity for Children: A Statement of Guidelines. Reston, Virginia.

5. Dubé, L. y Menon, K. (1998). Why would certain types of in-process negative emotions increase post-purchase consumer satisfaction with services? En T.A. Swartz, D.E. Bowen y S. Brown (eds.): Advances in service marketing and management (vol. 7, pp. 131-158). Greenwich: JAI Press.

6. Grönroos, C. (1982). Strategic Management and Marketing in the Ser- 
vice Sector. Swedish School of Economics and Business Administration. Sweden: Helsingfors.

7. Gwinner, K., Gremler, D.D. y Bitner, M.J. (1998). Relational benefits in services industries: The customer's perspective. Journal of the Academy of Marketing Science, 26, 101-114.

8. Hochschild, A. R. (1983). The managed heart: The commercialization of human being. Berkeley: University of California Press.

9. Malina, R. M. y Bouchard, C. (1991). Somatic growth. In R. M. Malina y C. Bouchard (Eds.), Growth, maduration, and physical activity (pp. 39-64), Champaign, IL: Human Kinetics.

10. Mañas, M.A., Giménez, G., Muyor, J.M., Martínez-Tur, V., y Moliner, C. (2008). Los tangibles como predictores de la satisfacción del usuario en servicios deportivos. Psicothema, 20, 243-248.

11. Martínez-Tur, V., Peiró, J.M., y Ramos, J. (2005). Linking situational constraints to customer satisfaction in a service environment. Applied Psychology: An International Review, 54, 25-36.

12. Morales-Sánchez, V. (2003). Evaluación psicosocial de la calidad en servicios municipales deportivos: aportaciones desde el análisis de variabilidad. Málaga: SPICUM.

13. Morales-Sánchez, V., Pérez-López, R y Anguera, M.T. (2014). Tratamiento metodológico de la observación indirecta en la gestión de organizaciones deportivas. Revista de Psicología del Deporte, 23(1), 201-207.

14. Pérez-López, R., Morales-Sánchez, V., Anguera, M.T. y HernándezMendo, A. (en prensa). Hacia la calidad de servicio emocional en organizaciones deportivas orientadas a la población infantil: un análisis cualitativo. Revista Iberoamericana de Psicología del Ejercicio y el Deporte.

15. Morales-Sánchez, V., Hernández-Mendo, A. y Blanco-Villaseñor, A. (2005). Evaluación de la calidad en los programas de actividad física. Psicothema, 17(2), 311-317.

16. Morales-Sánchez, V., Hernández-Mendo, A. y Blanco Villaseñor, A. (2009). Evaluación de la calidad en organizaciones deportivas: adaptación del modelo SERVQUAL. Revista de Psicología del Deporte, 18(2), 137-150.

17. Morris, A. J. y Fieldman, D. C. (1997). Managing emotions in the workplace. Journal of Managerial Issues, 9, 257-274.

18. Ntoumanis, N. (2001). A self-determination approach to the understanding of motivation in physical education. British Journal of Educational Psychology, 71, 225-242.
19. Parasuraman, A., Zeithaml, V. y Berry, L. (1985). A Conceptual Model of Service Quality and its Implications for Future Research. Journal of Marketing, 49, 41-50.

20. Parasuraman, A., Zeithaml, V. y Berry, L. (1988). SERVQUAL: A muliple-item scale for measuring consumer perceptions of service quality. Journal of Retailing, 64 (1), 12-40.

21. Parasuraman, A., Zeithaml, V y Berry, L. (1993). More on improving service quality measurement. Journal of Retailing, 69 (1), 140-147.

22. Parasuraman, A., Zeithaml, V. y Berry, L. (1994). Alternative Scales for Measuring Service Quality: A comparative assessment based on psychometric and diagnostic critera. Journal of Retailing, 70, 201-230.

23. Parasuraman, B.A. (1999). El papel de la tecnología en la prestación de servicios y el logro de la Excelencia en el Marketing. España: EOI y Fundación Airtel.

24. Price, L.L., Arnould, E.J. y Tierney, P. (1995). Going to extremes: Managing service encounters and assessing provider performance. Journal of Marketing, 59, 83-97.

25. Richard, M.D. y Sundaram, D.S. (1994). A model of lodging repeat choice intentions. Annals of Tourism Research, 21, 745-755.

26. Rothman KJ, Michels KB, Baum M. (2000). For and against: Declaration of Helsinki should be strengthened. British Medical Journal, 321, 442-445.

27. Sánchez-Hernández, R., Martínez-Tur, V., Peiró, J. M. y Ramos, J. (2009). Testing a hierarchical and integrated model of quality in the service sector: functional, relational, and tangible dimensions. Total Quality Management, 20(11),1173-1188.

28. Sasser, W., Olsen, R. P. y Wyckoff, D. D. (1978). Management of service operations: text and cases. Boston: Allyn \& Bacon.

29. Zapf, D., Vogt, C., Seifert, C., Mertini, y Isic, A. (1999). Emotion work as a source of stress: The concept and development of an instrument. European Journal of Work and Organizational Psychology, 8, 371-400.

30. Zeithaml, V.A., Parasuraman, A., y Berry, L.L. (1993). Calidad total en la gestión de los servicios. Cómo lograr el equilibrio entre las percepciones $y$ las expectativas de los consumidores. Madrid: Díaz de Santos.

31. Weinberg, R.S., y Gould, D. (1996). Fundamentos de psicología del deporte y el ejercicio físico. Barcelona: Ariel. 